\title{
Zentrale Begriffe im Kontext der Reallaborforschung
}

\author{
Annika Arnold \& Felix M. Piontek
}

Die Debatte um den Nutzen von Transdisziplinarität, transformativer Forschung und Reallaboren für eine gesellschaftliche Transformation und die damit verbundenen Herausforderungen mit Blick auf die Rolle der Wissenschaft wird derzeit in der Wissenschaft und auch darüber hinaus lebhaft geführt.

Bereits 1992 bezeichnete der Philosoph Jürgen Mittelstraß Transdisziplinarität als einen Weg, Wissen und Forschung mit lebensweltlichen Problemen in lösender Absicht zu verbinden. Die Spezialisierung der Disziplinen führt dazu, dass die einzelnen Wissenschaften gesellschaftliche Problemlagen, die nicht im jeweiligen disziplinären Gewand daherkommen, nicht oder erst sehr spät erkennen; eine inter- und transdisziplinäre Forschungsperspektive kann helfen, diese disziplinäre Eindimensionalität zu überwinden (Jaeger und Scheringer 1998). Mit der Postulierung des Formats Reallabor ist ein Baustein hinzugekommen, der in der Perspektive seiner Vertreter(innen) durch den damit einhergehenden transformativen Anspruch über bisherige transdisziplinäre Forschung hinausweist (Schneidewind und Singer-Brodowski 2015; Grunwald und Wagner 2015). Besonders dieser Anspruch wird kontrovers diskutiert: So attestieren Jahn und Keil (2016) dem Format Reallabor letztlich lediglich die Fortführung transdisziplinärer Forschungsprozesse mit anderen Mitteln, sie stellen also Reallabore als neuen Forschungsmodus infrage. Ein weiterer vorgetragener Kritikpunkt an transformativer Forschung stellt die Rolle der Wissenschaft ins Zentrum und fragt, welche Auswirkungen die Orientierung an gesellschaftlich normativen Zielvorgaben nach sich zieht (Strohschneider 2014).

Im Folgenden soll jedoch nicht die hier angerissene Debatte dargestellt und diskutiert werden. Vielmehr sollen zentrale Begriffe aus dem Kontext der Reallaborforschung aufgegriffen, erläutert und zueinander in Beziehung gesetzt werden. Dabei wird nicht der Anspruch auf eine allgemeingültige Definition erhoben. Stattdessen soll eine Kontextualisierung und Interpretation geboten werden, wobei der Kontext im Vordergrund steht, der für die Reallabore prägend war, deren Arbeit und Erfahrungen die Grundlage für das vorliegende Buch bildet. So haben 
Leser(innen) schnellen Zugriff auf Erklärungen unbekannter bzw. kontextspezifischer Begriffe. Der Beitrag weist aber auch über diesen Kontext hinaus und schafft damit die Möglichkeit, zentrale Themen und Begriffe der Debatte in ihren Grundzügen kennenzulernen. Er fungiert also auch als eine Handreichung insbesondere für diejenigen Leser(innen), die sich erstmalig der Reallaborforschung nähern.

Die gewählten Begriffe stammen überwiegend aus dem deutschsprachigen Raum und beziehen sich auf den Förderkontext der Reallabore in Baden-Württemberg, der „BaWü-Labs“. In zwei Förderlinien wurden durch das Ministerium für Wissenschaft, Forschung und Kunst Baden-Württemberg (MWK) ab 201514 BaWü-Labs eingerichtet (MWK 2013). ${ }^{1}$ Das in diesem Beitrag entfaltete Verständnis der diskutierten Begriffe spiegelt diesen Kontext, der den Autor(inn)en der im Buch versammelten Beiträge gemeinsam ist.

Folgende Begriffe und Themen werden im Text behandelt:

1) Transformative Forschung

2) Transdisziplinarität

3) Reallabor, Realexperiment

4) Akteursvielfalt

5) System-, Ziel- und Transformationswissen

6) Nachhaltigkeit als normatives Ziel der BaWü-Labs

\section{Transformative Forschung}

Transformative Forschung wird diskutiert und postuliert im Kontext von Überlegungen zur Rolle der Wissenschaft in einer Gesellschaft, die vor komplexen Problemlagen steht. In ihrem 2011 veröffentlichten Gutachten riefen die Mitglieder des Wissenschaftlichen Beirats der Bundesregierung Globale Umweltveränderungen (WBGU) dazu auf, eine neue Form wissenschaftlichen Arbeitens zu entwickeln: die transformative Forschung (Grunwald 2015; Schneidewind 2015; WBGU 2011). Diese steht in direkter Beziehung zur Transformationsforschung. Letztere nimmt sich den gesellschaftlichen Transformationsprozess zum Forschungsgegenstand, betrachtet also Transformationsprozesse hinsichtlich hemmender und fördernder Bedingungen, hinsichtlich ihrer Ausprägungen und ihres

1 Für Informationen zu den Förderlinien „Reallabore“ und „Reallabore Stadt“ siehe https://mwk.baden-wuerttemberg.de/de/forschung/forschungspolitik/wissenschaft-fuernachhaltigkeit/reallabore/ (zugegriffen am 07.02.2018). 
Verlaufs. Zentral ist dabei ein analytischer, objektiver Blick auf den Forschungsgegenstand.

In Abgrenzung hierzu nimmt transformative Forschung eine aktive Rolle als Unterstützerin von Transformationsprozessen ein, ,indem sie Umbauprozesse durch spezifische Innovationen in den relevanten Sektoren befördert. Sie unterstützt Transformationsprozesse konkret durch die Entwicklung von Lösungen sowie technischen und sozialen Innovationen“ (WBGU 2011, S. 374; s. auch Krainer und Winiwarter 2016). Transformative Forschung kann als normative Forschung beschrieben werden (Dubielzig und Schaltegger 2004), da sie auf eine an einer spezifischen Norm orientierte Veränderung gesellschaftlicher Strukturen und Kulturen abzielt (Schneidewind und Singer-Brodowksi 2014). Das Format Reallabor beinhaltet das Verfolgen transformativer Ziele und reiht sich damit in transformative Forschung ein (s. auch Beecroft et al. 2018).

\section{Transdisziplinarität}

Transdisziplinarität stellt die grundlegende Forschungsausrichtung in Reallaboren dar (Parodi 2016a et al., S. 285), um den geforderten Schritt vom Wissen zum Handeln zu gehen (Wagner und Grunwald 2015, S. 26). Die gesellschaftliche Relevanz der Probleme, die in Reallaboren behandelt werden, erfordert eine Verankerung des Forschungsprozesses unmittelbar in der Gesellschaft, um zu tragfähigen Lösungen zu gelangen.

Transdisziplinäre Forschung wird hier verstanden als eine Forschung, die sich lebensweltlicher Problemlagen annimmt, also solcher, die nicht einem reinen wissenschaftlichen Erkenntnisinteresse entspringen. Entsprechend ist die Integration von wissenschaftlichem und praktischem Wissen ein zentrales Versprechen transdisziplinärer Forschung. Es soll dadurch eingelöst werden, „dass disziplinäre Grenzen überwunden werden und alltagspraktisches Wissen von Praxisakteuren in den Produktionsprozess wissenschaftlicher Erkenntnis einbezogen wird" (Truffer 2007, S. 41). Eine solche Herangehensweise greift dort, wo

- der vorhandene Wissensbestand unsicher ist,

- die Problemlagen in ihren gesellschaftlichen Implikationen zunächst noch ausgehandelt werden müssen und

- wo angemessene Lösungswege erst noch zu identifizieren sind (vgl. hierzu Pohl und Hirsch-Hadorn 2008, S. 4f.; Burger und Kamber 2003, S. 44).

In einer transdisziplinären Herangehensweise umfasst der Einbezug also den gesamten Forschungsprozess, d. h. von der Erfassung der Problemlage über die Konstruktion möglicher Lösungen bis hin zur Erarbeitung wissenschaftlicher 
Erkenntnisse. Wissenschaft und Praxis arbeiten damit bereits bei der Identifikation der Probleme, die angegangen werden sollen, eng zusammen.

\section{Reallabor, Realexperiment}

Reallabore stellen ein neues Modell transdisziplinärer Forschung dar. Sie untersuchen, ,wie transdisziplinäre Forschung Anknüpfung an die gesellschaftlichen Akteure herstellt und biete[n] dafür ein setting an, das einen Rahmen für transdisziplinäre Prozesse bietet" (Ukowitz 2017, S. 10). Dieser Ansatz adressiert also die Beziehung transdisziplinärer Forschung zu ihrem gesellschaftlichen Kontext, z. B. Siedlungskontexte, Regionen, Branchen oder Institutionen (Wagner und Grunwald 2015, S. 27). Damit weist er über die abstrakt-prozessuale Ebene anderer Modelle transdisziplinärer Forschung hinaus, die beschreiben, „wie transdisziplinäre (Forschungs-)Prozesse idealtypisch ablaufen und wie inter- und transdisziplinäre Perspektiven- und Wissensintegration erfolgen" (Ukowitz 2017, S. 10). Reallabore bilden einen Forschungsansatz, durch den ein Beitrag zur Lösung eines gesellschaftlich relevanten Problemfelds geleistet werden soll - sie beziehen sich also auf konkrete soziale Kontexte und arbeiten transdisziplinär und transformativ.

Reallabore zeichnen sich entsprechend dadurch aus, dass mit verschiedensten Akteuren an verschiedenen Möglichkeiten der Transformation geforscht wird (Schäpke et al. 2017) und so Wissen für die Transformation erarbeitet wird (,vom Wissen zum Handeln“, MWK 2013, S. 31). In einem solchen Forschungsaufbau werden Lösungsansätze raumbezogen erarbeitet, wobei die Definition des passenden Raumbezuges aus dem einzelnen Reallabor heraus entsteht (s. das entsprechende Designprinzip in Beecroft et al. 2018). Entscheidend ist, dass bei der Erarbeitung eines Lösungsansatzes diejenigen Akteure einbezogen werden, die zu dem behandelnden Problem in Beziehung stehen. Der Begriff „Labor“ kann jedoch auch Irritationen hervorrufen, da er stark mit naturwissenschaftlicher Forschung verbunden ist. Im Reallaborkontext weist dieser Begriff zumeist eher auf die Forschungsinfrastruktur hin, also auf ,eine Forschungseinrichtung, die - ana$\log \mathrm{zu}$ einem ingenieur- oder naturwissenschaftlichen Laboratorium - einen zweckdienlichen Rahmen, den Laborraum“ (Parodi et al. 2016a, S. 285) zur Verfügung stellt. Reallabore „bieten ein institutionalisiertes Setting als Rahmen für Experimente" (Wagner und Grunwald 2015, S. 26).

Solche Experimente können in Reallaboren als Realexperimente auftreten. Diese Form des Experiments bietet die Möglichkeit, Lösungswege für gesellschaftliche Problemlagen zu erproben, die Gegenstand des jeweiligen Reallabors sind. Realexperimente sind ein möglicher und typischer, aber kein notwendiger Teil eines 
Tabelle 1: Unterscheidung Realexperiment und naturwissenschaftliches Experiment.

\begin{tabular}{|c|c|c|}
\hline & Realexperiment & Experiment \\
\hline Primäres Ziel & $\begin{array}{l}\text { Schaffung von Wissen zur Lösung } \\
\text { gesellschaftlicher Problemlagen }\end{array}$ & $\begin{array}{l}\text { Schaffung von Wissen zur } \\
\text { Lösung wissenschaftlicher } \\
\text { Probleme }\end{array}$ \\
\hline Dokumentation & $\begin{array}{l}\text { Dokumentation dem Thema, den } \\
\text { Akteuren und der Intervention } \\
\text { entsprechend und wie unter den } \\
\text { jeweiligen Bedingungen umsetzbar }\end{array}$ & $\begin{array}{l}\text { umfassende Dokumentation } \\
\text { nach wissenschaftlichen } \\
\text { Standards }\end{array}$ \\
\hline $\begin{array}{l}\text { Experimentelles } \\
\text { Umfeld }\end{array}$ & $\begin{array}{l}\text { Durchführung in einem lebenswelt- } \\
\text { lichen ,Raum' }\end{array}$ & $\begin{array}{l}\text { Durchführung im isolierten } \\
\text { Labor }\end{array}$ \\
\hline Durchführende & $\begin{array}{l}\text { alle Akteure eines Reallabors und } \\
\text { Angehörige des jeweiligen lebens- } \\
\text { weltlichen ,Raums' }\end{array}$ & wissenschaftliches Personal \\
\hline $\begin{array}{l}\text { Wiederhol- } \\
\text { barkeit }\end{array}$ & $\begin{array}{l}\text { kann aufgrund seines Kontextes } \\
\text { (beständiger gesellschaftlicher } \\
\text { Wandel, keine kontrolliert herstell- } \\
\text { baren Laborbedingungen) nicht } \\
\text { exakt wiederholt werden }\end{array}$ & $\begin{array}{l}\text { muss zur wissenschaftlichen } \\
\text { Validität wiederholbar sein }\end{array}$ \\
\hline Reliabilität & $\begin{array}{l}\text { unkontrollierbare Bedingungen des } \\
\text { Experimentes lassen kaum Aussa- } \\
\text { gen zur Verlässlichkeit der Messun- } \\
\text { gen zu }\end{array}$ & $\begin{array}{l}\text { hohes Maß der Verlässlich- } \\
\text { keit der Messungen not- } \\
\text { wendig (Stabilität, Konsis- } \\
\text { tenz, Äquivalenz) }\end{array}$ \\
\hline
\end{tabular}

Reallabors; sie sind aber eine spezielle Form des Forschungssettings und sollen daher hier betrachtet werden. Der Begriff „Realexperiment“ knüpft an den naturwissenschaftlichen Begriff eines Experimentes an: Letzteres beschreibt eine Intervention, zeichnet sich durch kontrollierte Bedingungen der Durchführung, Einbindung in einen wissenschaftlichen (theoretischen und/oder methodischen) Kontext, umfassende Dokumentation und die Schaffung von Wissen als primärem Ziel aus.

Beide Formen, Realexperiment und naturwissenschaftliches Experiment, stellen Interventionen in einem System dar. Sie unterscheiden sich jedoch in bestimmten Aspekten (Tab. 1 führt diese differenzierenden Charakteristika auf). Bei einem Realexperiment findet die Intervention grundsätzlich im öffentlichen Raum statt und ist grundsätzlich offen gegenüber der Beteiligung der Öffentlichkeit (wobei es von der Thematik eines Reallabors abhängt, was jeweils als Öffentlichkeit gilt). Dadurch entfällt die Möglichkeit kontrollierbarer bzw. kontrolliert veränderbarer Laborbedingungen, unter denen das Experiment durchgeführt wird. Im Einklang mit der Zielstellung transdisziplinärer und transformativer Forschung 
wird mit Realexperimenten die Absicht verfolgt, einen Beitrag zur Lösung gesellschaftlicher Problemlagen zu leisten, wohingegen naturwissenschaftliche Experimente zuerst einmal wissenschaftlichen Erkenntnisinteressen dienen.

\section{Akteursvielfalt}

Wie bereits im Kapitel 2 ausgeführt, spielen außerwissenschaftliche Akteure eine bedeutende Rolle im transdisziplinären Forschungsprozess (z. B. Jaeger und Scheringer 1998, S. 11; Pohl und Hirsch-Hadorn 2008, S. 7) und damit auch in der Forschung in Reallaboren. Zur Kategorisierung der beteiligten Akteure wird im Folgenden zwischen ,Wissenschaft" und ,Praxis' unterschieden. ,Wissenschaft ' beschreibt das gesamte System von akademischer Wissenserarbeitung und -bereitstellung, inklusive der theoretischen Einbettung von empirischen Daten. Der,Wissenschafts-Bereich' stellt also das wissenschaftlich arbeitende Personal. ,Praxis' bezieht sich auf den lebensweltlichen Bereich, in den die zu behandelnde Problemlage fällt. Auch wenn die Akteure in diesem Bereich über wissenschaftliches Wissen verfügen, bringen sie in erster Linie Erfahrungswissen aus der Praxis in ein Reallabor ein.

Die enge Verknüpfung dieser beiden Bereiche Wissenschaft und Praxis sowie die Vielzahl von gesellschaftlichen Implikationen der Problemlagen, die in einem Reallabor betrachtet werden, zieht eine hohe Akteursvielfalt in einem Reallabor nach sich. Die Praxispartner eines Reallabors können aus vielen unterschiedlichen Kontexten stammen (z. B. organisierte Zivilgesellschaft, lokale Gemeinschaft, kommunale (auch universitäre) Verwaltung, Wirtschaftsunternehmen, Politik, Kultur, Bildung). Die Zusammensetzung der Praxispartner hängt von der thematischen, sozialräumlichen und zeitlichen Ausrichtung eines Reallabors ab (s. auch Beecroft et al. 2018; Eckart et al. 2018). Praxispartner können in unterschiedlichem Ausmaß in die Forschungsprozesse eines Reallabors eingebunden sein: Sie können (gleichberechtigte) Mitglieder des Reallaborteams über den gesamten Zeitraum hinweg sein oder als „externe Beteiligte“ inhaltlich und/oder zeitlich punktuell einen oder mehrere substantielle Beiträge zu den Forschungsfragen leisten (vgl. Defila et al. 2006, S. 216f.). Die Kontexte, die einzelne Praxispartner in einem Reallabor vertreten, können sich zudem während des Durchführungszeitraumes ändern, bspw. wenn eine Gruppe aus der lokalen Gemeinschaft einen Verein gründet und fortan dem Reallabor in neuer, organisierter Form verbunden ist (s. zur Komplexität der Akteurskonstellation in Reallaboren auch Seebacher et al. 2018).

Innerhalb der Praxispartner lässt sich eine Untergruppe ausmachen: die der sogenannten „Pioniere des Wandels“ oder „Change Agents“. Solche Akteure spielen in manchen Reallaboren eine besondere Rolle. Ihnen wird zum einen zugeschrie- 
ben, dass sie eine Idee zur Veränderung eines Ist-Zustandes aus der Zivilgesellschaft in die Forschung tragen können (vgl. Kristof 2010). Zum anderen wird ihnen zugeschrieben, dass sie althergebrachte Perspektiven herausfordern und neue Wege der Veränderung vorschlagen könnten (WBGU 2011, S. 257). Die Ausrichtung des Veränderungsstrebens sollte dabei den Zielen des Reallabors entsprechen (s. hierzu Kapitel 6). „Pioniere des Wandels“ werden am Beginn einer gesellschaftlichen Transformation gesehen (Grießhammer und Brohmann 2015, S. 14, S. 40f.), wo sie als Promotoren (Kristof 2010) eine kritische Masse der Bevölkerung zur Veränderung (bspw. von Konsumroutinen) aktivieren wollen (Becker und Scheibler 2013, S. 2f.). „Pioniere des Wandels“ werden oft in der Zivilgesellschaft verortet, teils als Einzelakteure, teils als Verbünde, sie können sich aber natürlich auch in den Reihen von Unternehmen aus der Wirtschaft oder bei Akteuren der öffentlichen Hand finden.

\section{System-, Ziel- und Transformationswissen}

Reallabore leisten einen Beitrag zur Lösung gesellschaftlicher Problemlagen (z. B. Vernutzung natürlicher Ressourcen, Erhalt sozialer Strukturen), sie reichen von der Zielsetzung her also über ein rein wissenschaftlich motiviertes Erkenntnisinteresse hinaus. Damit müssen sie auch eine andere Art von Wissen generieren als solches, das im selbstbezogenen Wissenschaftsdiskurs oft primär gefordert ist. Reallabore zielen auf die Erzeugung von drei unterschiedlichen Wissensarten ab (CASS und ProClim- 1997, S. 15):

- „Wissen darüber, was ist: Systemwissen über Strukturen und Prozesse, Variabilität usw.“

- „Wissen darüber, was sein und was nicht sein soll: Zielwissen, d. h. Bewertung von Ist-Zustand, Prognosen und Szenarien; Generierung von Grenzwerten, ,Leitbildern', ethischen Rahmenbedingungen, Visionen.“

- „Wissen darüber, wie wir vom Ist- zum Soll-Zustand gelangen: Transformationswissen, d. h. Erarbeitung von Wissen darüber, wie der Übergang vom Istzum Soll-Zustand gestaltet werden kann.“

Systemwissen stellt Antworten bereit auf die Frage nach der Entstehung und der weiteren Entwicklung von gesellschaftlichen Problemen, die mit dem jeweiligen Forschungsprozess adressiert werden sollen. Zielwissen geht der Frage nach, welcher Veränderungsbedarf besteht, welche Ziele erwünscht sind. Transformationswissen schließlich eruiert die Möglichkeiten, mit denen diese Ziele erreicht werden können (Pohl und Hirsch-Hadorn 2008). 
Diese Unterscheidung von Wissensarten ist allerdings idealtypisch zu verstehen, denn im Forschungsalltag lassen sich diese nicht unbedingt scharf trennen: „Die Produktion von Systemwissen, Zielwissen und Transformationswissen bildet selten eine klar abgetrennte Sequenz. Vielmehr fließt etwa bereits in die Analyse eines Problems viel explizites und implizites Ziel- und Transformationswissen mit ein, Zielwissen kann nicht ohne eine gewisse Vorstellung über Transformationsmöglichkeiten erarbeitet werden, und so weiter" (Truffer 2007, S. 42).

In Reallaboren wird Wissen in Zusammenarbeit zwischen Wissenschaft und Praxis und damit auch im Zusammenführen unterschiedlicher Wissensbestände erzeugt. Diese Wissensintegration bietet eine Möglichkeit auch für Prozesse des sozialen Lernens für eine gesellschaftliche Transformation (Schneidewind und Singer-Brodowski 2015; Wenger 1998). Ein solches Lernen ist dann gegeben, wenn alle beteiligten Akteure erstens voneinander lernen und zweitens das Gelernte in einen gesellschaftlichen Aushandlungsprozess einbringen. Reallabore eröffnen so die Möglichkeit eines „gesellschaftlichen Lernprozesses“, in dem Wissen für eine gesamtgesellschaftliche Transformation erarbeitet wird (Parodi et al. 2016b; zu den Bildungszielen von Reallaboren s. auch Beecroft et al. 2018).

\section{Nachhaltigkeit als normatives Ziel der BaWü-Labs}

Die normativen gesellschaftlichen Ziele, die einem Reallabor zugrunde liegen, können vielfältiger Natur sein und beeinflussen die Arbeit eines Reallabors, nicht jedoch die Arbeitsweise. Generell lässt sich festhalten, dass Reallabore auf gesellschaftliche Ziele ausgerichtet sein sollten, die gemeinwohlorientiert, ethisch begründet und gesellschaftlich legitimiert sind (s. dazu auch Defila und Di Giulio 2018).

Im Falle der BaWü-Labs konkretisiert sich diese Zielstellung in einer Orientierung auf den Themenkomplex Nachhaltigkeit (Eckart 2011; Grunwald und Kopfmüller 2012). Dieses Thema ist in der Forschungsförderung für die BaWüLabs vorgegeben: „Alle BaWü-Labs sollten konkrete gesellschaftliche Herausforderungen aufgreifen [und] sich an den spezifischen Nachhaltigkeitsfragen des Landes orientieren“" (MWK 2013, S. 37).

Damit kommt den BaWü-Labs eine inhärente Nachhaltigkeitsorientierung zu. Die „Brundtland-Definition“ von Nachhaltigkeit ${ }^{2}$ wird für das Land Baden-Württem-

2 ,Sustainable development meets the needs of the present without compromising the ability of future generations to meet their own needs" (Nachhaltig ist eine Entwicklung, ,, die den Bedürfnissen der heutigen Generation entspricht, ohne die Möglichkei- 
berg durch den Zusatz erweitert: „Nachhaltig handelt heißt, nicht auf Kosten von Menschen in anderen Regionen der Erde zu leben oder die Erfüllung der Bedürfnisse zukünftiger Generationen zu gefährden. Wirtschaftliche, soziale und ökonomische Aspekte sind dabei gleichermaßen zu berücksichtigen. Dabei bildet die Belastbarkeit der Erde und der Natur die absolute Grenze: Ein Rückgang an natürlichen Ressourcen, also der Abbau von Rohstoffen oder der Verlust natürlicher Lebensräume, kann nicht durch steigendes Kapital in einem der anderen Bereiche ausgeglichen werden“ (MUKE 2014, S. 8). Die Projekte aus den Förderlinien „Reallabore“ und „Reallabore Stadt" des MWK behandeln eine Vielzahl von Nachhaltigkeitsaspekten in unterschiedlicher Weise (s. für Kurzbeschreibungen und weiterführende Links Fn 1).

Die Nachhaltigkeitsstrategie des Landes Baden-Württemberg strebt an, die Rolle der Wissenschaft für eine nachhaltige Entwicklung und deren integrale Einbindung in die landesweiten Nachhaltigkeitsbemühungen (MWK 2013, S. 7) zu adressieren. Die Einrichtung von Reallaboren soll nach dieser Strategie einen wesentlichen Bestandteil zur Unterstützung von transdisziplinärer und transformativer Wissenschaft bilden (MWK 2013, S. 31).

\section{Dank}

Die Autorin und der Autor danken Richard Beecroft, Elke Häußler, Regina Rhodius, Ines-Ulrike Rudolph, Andreas Seebacher, Helena Trenks und Christina West für die Diskussionen über frühere Fassungen des Textes im Rahmen des internen Reviews. Insbesondere möchten sie zwei anonymen externen Gutachter(inne)n für die konkreten Hinweise zur Verbesserung des Textes danken. Schließlich danken der Autor und die Autorin den beiden Herausgebenden, Rico Defila und Antonietta Di Giulio, für ihre Rückmeldungen zum Text.

\section{Literatur}

Becker, S., \& Scheibler, U. (2013). Netzwerke für den „Wandel“: Soziale Netzwerke und die Beziehungskultur von Change Agents am Beispiel des Göttinger Pilotprojekts „PermaKulturRaum“. In J. Friedrich, A. Halsband \& L. Minkmar (Hrsg.), Tagungsband zur Fachtagung Biodiversität und Gesellschaft. Gesellschaftliche Dimensionen von Schutz und Nutzung biologischer Vielfalt (S. 33-43). Göttingen: Göttingen University Press.

ten künftiger Generationen zu gefährden, ihre eigenen Bedürfnisse zu befriedigen und ihren Lebensstil zu wählen") (Brundtland 1987, S. 41). 
Beecroft, R., Trenks, H., Rhodius, R., Benighaus, C., \& Parodi, O. (2018). Reallabore als Rahmen transformativer und transdisziplinärer Forschung: Ziele und Designprinzipien. In R. Defila \& A. Di Giulio (Hrsg.), Transdisziplinär und transformativ forschen. Eine Methodensammlung (S. 75-100). Wiesbaden: Springer VS.

Brundtland, G. H. (1987). Report of the World Commission on environment and development: „our common future“. United Nations.

Burger, P., \& Kamber, R. (2003). Cognitive Integration in Transdisciplinary Science. Knowledge as a Key Notion. Issues in Integrative Studies, 21, (S. 43-73).

CASS \& ProClim- (1997). Forschung zu Nachhaltigkeit und Globalem Wandel - Wissenschaftspolitische Visionen der Schweizer Forschenden. Bern: ProClim-/SANW. https://naturwissenschaften.ch/service/publications/75640-visionen-der-forschenden. Zugegriffen am 21.01.2018.

Defila, R., \& Di Giulio, A. (2018). Reallabore als Quelle für die Methodik transdisziplinären und transformativen Forschens - eine Einführung. In R. Defila \& A. Di Giulio (Hrsg.), Transdisziplinär und transformativ forschen. Eine Methodensammlung (S. 935). Wiesbaden: Springer VS.

Defila, R., Di Giulio, A., \& Scheuermann, M. (2006). Forschungsverbundmanagement. Handbuch für die Gestaltung inter- und transdisziplinärer Projekte. Zürich: vdf Hochschulverlag an der ETH Zürich.

Dubielzig, F., \& Schaltegger, S. (2004). Methoden transdisziplinärer Forschung und Lehre: Ein zusammenfassender Überblick. Lüneburg. http://www2.leuphana.de/ umanagement/csm/content/nama/downloads/download_publikationen/49-8download version.pdf. Zugegriffen am 14.02.2018.

Eckart, F. (2011). Theorie der Nachhaltigkeit: Rechtliche, ethische und politische Zugänge - am Beispiel von Klimawandel, Ressourcenknappheit und Welthandel. Baden-Baden: Nomos.

Eckart, J., Ley, A., Häußler, E., \& Erl, Th. (2018). Leitfragen für die Gestaltung von Partizipationsprozessen in Reallaboren. In R. Defila \& A. Di Giulio (Hrsg.), Transdisziplinär und transformativ forschen. Eine Methodensammlung (S. 105-135). Wiesbaden: Springer VS.

Grießhammer, R., \& Brohmann, B. (2015). Wie Transformationen und gesellschaftliche Innovationen gelingen können. Umweltbundesamt (Hrsg.). Dessau-Roßlau. https:// www.umweltbundesamt.de/sites/default/files/medien/376/publikationen/wie_transfor mationen_und_gesellschaftliche_innovationen_gelingen_koennen.pdf. Zugegriffen am 09.02.2018.

Grunwald, A. (2015). Transformative Wissenschaft - eine neue Ordnung im Wissenschaftsbetrieb? GAIA, 24 (1), (S. 17-20).

Grunwald, A., \& Kopfmüller, J. (2012). Nachhaltigkeit. Frankfurt a. M., New York: Campus.

Jaeger, J., \& Scheringer, M. (1998). Transdisziplinarität: Problemorientierung ohne Methodenzwang. GAIA, 7 (1), (S. 10-25). 
Jahn, T., \& Keil, F. (2016). Reallabore im Kontext transdisziplinärer Forschung. GAIA, 25 (4), (S. 247-252).

Krainer, L., \& Winiwarter, V. (2016). Die Universität als Akteurin der transformativen Wissenschaft: Konsequenzen für die Messung der Qualität transdisziplinärer Forschung. GAIA, 25 (2), (S. 110-116). doi: 10.14512/gaia.25.2.11.

Kristof, K. (2010). Wege zum Wandel. Wie wir gesellschaftliche Veränderungen erfolgreicher gestalten können. München: OEKOM.

Mittelstraß, J. (1992). Auf dem Wege zur Transdisziplinarität. GAIA, 1 (5), (S. 250).

MUKE (Ministerium für Umwelt, Klima und Energiewirtschaft Baden-Württemberg) (2014). Nachhaltigkeitsstrategie Baden-Württemberg. Stuttgart. http://www.nach haltigkeitsstrategie.de/fileadmin/Downloads/N-Service/publikationen/N-Strategie Broschuere_web.pdf. Zugegriffen am 09.02.2018.

MWK (Ministerium für Wissenschaft, Forschung und Kunst Baden-Württemberg) (Hrsg.) (2013). Wissenschaft für Nachhaltigkeit. Herausforderung und Chance für das badenwürttembergische Wissenschaftssystem. Stuttgart. https://www.baden-wuerttemberg. de/fileadmin/redaktion/dateien/PDF/Broschüre_Wissenschaft_für_Nachhaltigkeit.pdf. Zugegriffen am 09.02.2018.

Parodi, O., Albiez, M., Beecroft, R., Meyer-Soylu, S., Quint, A., Seebacher, A., \& Waitz, C. (2016a). Das Konzept „Reallabor“ schärfen: Ein Zwischenruf des Reallabor 131: KIT findet Stadt. GAIA, 25 (4), (S. 284-285). doi: 10.14512/gaia.25.4.11.

Parodi, O., Beecroft, R., Albiez, M., Quint, A., Seebacher, A., Tamm, K., \& Waitz, C. (2016b). Von „Aktionsforschung“ bis „Zielkonflikte“ - Schlüsselbegriffe der Reallaborforschung. Technikfolgenabschätzung - Theorie und Praxis, 25 (3), (S. 9-18).

Pohl, C., \& Hirsch Hadorn, G. (2008). Gestaltung transdisziplinärer Forschung. Sozialwissenschaften und Berufspraxis, 31 (1), (S. 5-22).

Schäpke, N., Stelzer, F., Bergmann, M., Singer-Brodowski, M., Wanner, M., Caniglia, G., \& Lang, D. J. (2017). Reallabore im Kontext transformativer Forschung. Ansatzpunkte zur Konzeption und Einbettung in den internationalen Forschungsstand. (No. 1/2017). Leuphana Universität Lüneburg, Institut für Ethik und Transdisziplinäre Nachhaltigkeitsforschung.

Schneidewind, U. (2015). Transformative Wissenschaft - Motor für gute Wissenschaft und lebendige Demokratie. GAIA, 24 (2), (S. 88-91). doi: 10.14512/gaia.24.2.5.

Schneidewind, U., \& Singer-Brodowski, M. (2014). Transformative Wissenschaft. Klimawandel im deutschen Wissenschafts- und Hochschulsystem. 2. Aufl., Marburg: Metropolis.

Schneidewind, U., \& Singer-Brodowski, M. (2015). Vom experimentellen Lernen zum transformativen Experimentieren - Reallabore als Katalysator für eine lernende Gesellschaft auf dem Weg zu einer Nachhaltigen Entwicklung. Zeitschrift für Wirtschaftsund Unternehmensethik, 16 (1), (S. 10-23). 
Seebacher, A., Alcántara, S., \& Quint, A. (2018). Akteure in Reallaboren - Reallabore als Akteure. In R. Defila \& A. Di Giulio (Hrsg.), Transdisziplinär und transformativ forschen. Eine Methodensammlung (S. 155-159). Wiesbaden: Springer VS.

Strohschneider, P. (2014). Zur Politik der Transformativen Wissenschaft. In A. Brodocz, D. Herrmann, R. Schmidt, D. Schulz, \& J. Schulze Wessel (Hrsg.), Die Verfassung des Politischen (S. 175-192). Wiesbaden: Springer Fachmedien. doi: 10.1007/978-3-65804784-9_10.

Truffer, B. (2007). Wissensintegration in transdisziplinären Projekten. Flexibles Rollenverständnis als Schlüsselkompetenz für das Schnittstellenmanagement. GAIA, 16 (1), (S. 41-45).

Ukowitz, M. (2017). Transdisziplinäre Forschung in Reallaboren. Ein Plädoyer für Einheit in der Vielfalt. GAIA, 26 (1), (S. 9-12).

Wagner, F., \& Grunwald, A. (2015). Reallabore als Forschungs- und Transformationsinstrument: Die Quadratur des hermeneutischen Zirkels. GAIA, 24 (1), (S. 26-31).

WBGU (Wissenschaftlicher Beirat für Globale Umweltveränderungen) (2011). Welt im Wandel. Gesellschaftsvertrag für eine Große Transformation. Hauptgutachten 2011. Berlin: WBGU. Verfügbar unter: http://www.wbgu.de/hauptgutachten/hg-2011-Trans formation. Zugegriffen am 18.02.2018.

Wenger, E. (1998). Communities of practice: learning, meaning, and identity. Cambridge: Cambridge University Press.

Open Access Dieses Kapitel wird unter der Creative Commons Namensnennung 4.0 International Lizenz (http://creativecommons.org/licenses/by/4.0/deed.de) veröffentlicht, welche die Nutzung, Vervielfältigung, Bearbeitung, Verbreitung und Wiedergabe in jeglichem Medium und Format erlaubt, sofern Sie den/die ursprünglichen Autor(en) und die Quelle ordnungsgemäß nennen, einen Link zur Creative Commons Lizenz beifügen und angeben, ob Änderungen vorgenommen wurden.

Die in diesem Kapitel enthaltenen Bilder und sonstiges Drittmaterial unterliegen ebenfalls der genannten Creative Commons Lizenz, sofern sich aus der Abbildungslegende nichts anderes ergibt. Sofern das betreffende Material nicht unter der genannten Creative Commons Lizenz steht und die betreffende Handlung nicht nach gesetzlichen Vorschriften erlaubt ist, ist für die oben aufgeführten Weiterverwendungen des Materials die Einwilligung des jeweiligen Rechteinhabers einzuholen. 